\title{
An Improved Treatment of Optics in the Lindquist-Wheeler Models
}

\author{
Timothy Clifton $*$ and Pedro G. Ferreird ${ }^{\dagger}$ \\ Department of Astrophysics, University of Oxford, UK. \\ Kane O'Donnel围 \\ Department of Physics and Astronomy, University of Canterbury, New Zealand.
}

\begin{abstract}
We consider the optical properties of Lindquist-Wheeler (LW) models of the Universe. These models consist of lattices constructed from regularly arranged discrete masses. They are akin to the Wigner-Seitz construction of solid state physics, and result in a dynamical description of the largescale Universe in which the global expansion is given by a Friedmann-like equation. We show that if these models are constructed in a particular way then the redshifts of distant objects, as well as the dynamics of the global space-time, can be made to be in good agreement with the homogeneous and isotropic Friedmann-Lemaître-Robertson-Walker (FLRW) solutions of Einstein's equations, at the level of $\lesssim 3 \%$ out to $z \simeq 2$. Angular diameter and luminosity distances, on the other hand, differ from those found in the corresponding FLRW models, while being consistent with the 'empty beam' approximation, together with the shearing effects due to the nearest masses. This can be compared with the large deviations found from the corresponding FLRW values obtained in a previous study that considered LW models constructed in a different way. We therefore advocate the improved LW models we consider here as useful constructions that appear to faithfully reproduce both the dynamical and observational properties of space-times containing discrete masses.
\end{abstract}

\section{INTRODUCTION}

In previous papers [1, 2] we have considered luminosity distances and redshifts in the (LW) models of the Universe [3]. These models treat space-time as a lattice of regular cells, each with a mass at their center. The geometry inside a given cell is then calculated under the assumption that the influences of all masses external to that cell are approximately spherically symmetric. This is the direct gravitational analogue of the Wigner-Seitz construction from electromagnetism [4].

Unlike the models constructed by Wigner and Seitz, however, the LW models (without a cosmological constant) are necessarily dynamical. This is due to the single charge available in gravitational theory, that causes a non-zero derivative of the gravitational field normal to the boundaries of each cell. The resulting force then causes the cell boundaries to fall toward, or away from, the central mass of each cell, and makes the entire lattice dynamical. Within this model the phenomenon of an expanding universe is then an emergent one: The global cosmological dynamics result from gluing together the space-times around individual masses, each of which is well described locally as being static. These models do not require us to specify a 'background geometry' for the Universe, and therefore provide an opportunity to study the 'back-reaction' of structures on the global evolution of the Universe [5]. They also provide a simple model of an inhomogeneous universe, within which calculations can be readily performed.

\footnotetext{
*Electronic address: tclifton@astro.ox.ac.uk

${ }^{\dagger}$ Electronic address: pgf@astro.ox.ac.uk

‡Electronic address: odonnell.kane@gmail.com
}

Lattices constructed in a positively curved space with no cosmological constant were considered in the original work on this subject by Lindquist and Wheeler [3] . These models were shown to obey evolution equations identical to those of the spatially homogeneous and isotropic Friedmann-Lemaître-Robertson-Walker (FLRW) solutions of Einstein's equations with pressureless dust sources. Only the scale of the solutions to those equations was found to be different. This study was generalized to include lattices constructed in spatially flat and negatively curved spaces in [1, 6], and to include a cosmological constant in [2]. Both spatially flat and negatively curved models were also shown to obey the expected Friedmann equations. A further interesting extension of the original LW models was performed in [7], where lattices with only two cells were considered, and shown to exist as solutions of Einstein's equations only when $\Lambda \neq 0$ and when the masses are out of causal contact with each other.

Unfortunately, the LW models (with the exception of the 2 mass case) are not exact solutions of Einstein's equations, and the way that the global lattice is constructed is not entirely unambiguous. In their original paper, Lindquist and Wheeler discussed what they considered to be two reasonable methods of constructing such a structure; what they referred to as Condition I and Condition II in [3] (these conditions will be discussed in more detail in Section 【I). Both of these conditions give the same Friedmann equations, but lead to different predictions for the change in scale of the global dynamics. The optical properties of spatially flat models that obey Condition II were considered in [1, 2] [14]. In these papers detailed calculations of the redshifts and luminosity distances that would be seen by observers in these models were performed, both with and without a cosmological constant. Large deviations from the predictions of the 
corresponding quantities in FLRW models were found, and the effects that this could have on cosmological parameter estimation were considered [2]. This line of study was generalized to spatially curved models in [6].

Here we study the optical properties of spatially flat Lindquist-Wheeler models using Condition I of [3]. It has recently been shown that the change in scale predicted by positively curved LW models constructed using Condition I is in good keeping with the corresponding exact solutions of Einstein's equations, including back-reaction [9]. It has also been shown that spatially flat LW models constructed using Condition I provide a good description of the global evolution of space-time when their cells are much smaller than the Hubble radius, and much larger than the Schwarzschild radius of the central masses [8]. Condition II does not provide a good description of the space-time in either of these cases. We consider this to provide sufficient motivation to explore the optical properties of LW models that are constructed using Condition I, rather than those of the apparently less favorable Condition II that were studied in [1, 2].

In Section [I] we briefly describe the way in which the LW models are constructed, paying particular attention to the difference between Condition I and Condition II of [3]. In Section [II we then recap on how observational quantities such as redshifts and luminosity distances are calculated in these models. In Section IV we show the results of using Condition I when calculating distance measures and redshifts in the spatially flat models. It is found that deviations from the corresponding quantities in spatially flat FLRW models are typically $\lesssim 3 \%$ at $z \lesssim 1$, in stark contrast to the results found when using Condition II [1, 2]. In Section V we discuss these results, and their consequences for the validity and applicability of the LW construction as a model of the Universe. We conclude that the LW model provides a surprisingly accurate model of both the cosmological dynamics (including the back-reaction of structures) and optical properties of the space-times associated with regularly spaced discrete masses. The applicability of these ideas to more irregular configurations of matter remains to be demonstrated.

\section{THE MODEL}

The LW models are constructed by tiling spaces of constant curvature with regular polyhedra, and placing a mass $m$ at the center of each polyhedron. The geometry of the space-time within each cell is then calculated under the assumption that the influence of the external masses is spherically symmetric, and boundary spheres of constant Schwarzschild radial distance $r$ are allowed to follow geodesics of the resulting geometry. This results in the space-time around each mass being described as Schwarzschild-de Sitter geometry. By specifying a relation between the boundary spheres and the original polyhedra one then has a dynamical lattice model of the Universe.
In order to perform calculations one now requires measures of cosmological size and time. These are provided in the positively curved case by the radius of the lattice in an embedding Euclidean 4-space, and by the proper time $\tau$ along the trajectories of the boundary spheres [3]. These concepts were extended to the case of spatially flat and negatively curved lattices in [1]. The result is that these lattices obey the evolution equation

$$
\frac{\dot{a}_{L W}^{2}}{a_{L W}^{2}}=\frac{2 m}{a_{L W}^{3} f^{3}(\psi)}-\frac{k}{a_{L W}^{2}},
$$

where $a_{L W}=r / f(\psi)$ is the size of the lattice (in the original 3-space of constant curvature, to which the trajectories of the spherical boundaries are made to be orthogonal), $m$ is the 'bare mass' at the center of each cell, $k=1,0$ or -1 depending on the spatial curvature of the lattice, and over-dots denote differentiation with respect to $\tau$. The function $f(\psi)=\sin (\psi), \psi$ or $\sinh (\psi)$ for $k=1$, 0 or -1 , respectively, where $\psi$ is a constant (the angle subtended by a single spherical boundary at the origin of the embedding space when $k=1$ ).

The precise value of $\psi$ depends on the lattice that is being constructed (see Appendix A of [1] for all the possible tilings of constant curvature 3 -spaces with regular polyhedra), and the way in which the lattice cells are identified with boundary spheres. Lindquist and Wheeler suggested two possible conditions for achieving this:

Condition I: The boundary sphere shall occupy the same volume of the lattice as each cell.

Condition II: The boundary sphere associated with each mass shall just touch the boundary sphere of all its nearest neighbors.

These two possibilities are illustrated in Figure 1 The value of $\psi$ for each of the six possible lattices that can be constructed in a positively curved space are then given in Table IV of [3]. In a flat space the only possible lattice has $\psi=\left(\frac{6}{\pi}\right)^{\frac{1}{3}} \simeq 1.24$ under Condition I, and $\psi=1$ under Condition II. Throughout this paper we will refer to lattices constructed using Condition I as Type I, and those constructed using Condition II as Type II.

It has been shown in [8] that when the inter-mass separation is small compared to the Hubble scale, but large compared to the Schwarzschild radius of the masses, that Type I lattices accurately reproduce the large-scale dynamics of more accurate solutions to Einstein's equations. Using exact solutions, Type I lattices have also been shown in [9] to accurately predict the scale of the maximum of expansion of positively curved universes (including the predicted deviation from the corresponding FLRW solutions). Type II lattices have been shown to produce inaccurate results for the global dynamics of the space-time in both of these cases. 


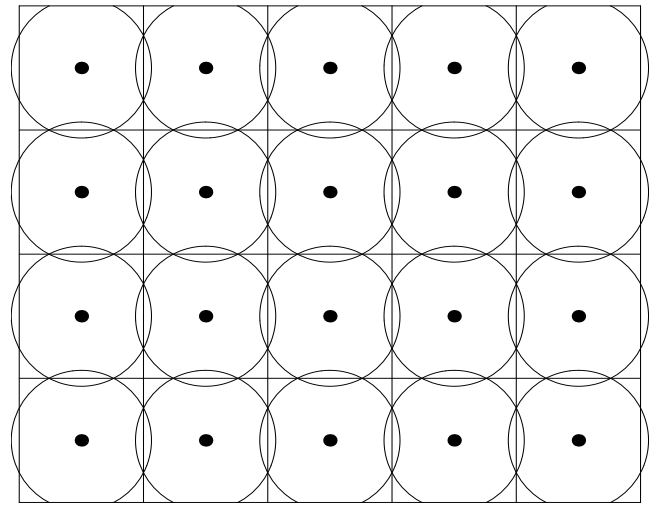

(a) Type I

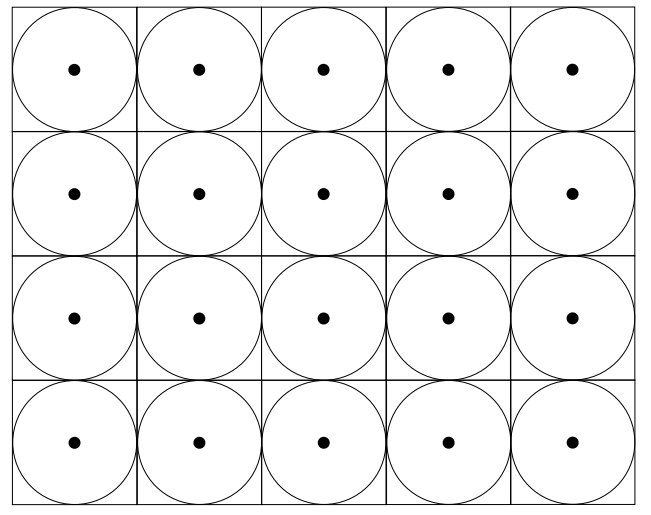

(b) Type II

FIG. 1: Illustration of Type I and Type II lattices, constructed using Condition I and Condition II.

\section{OPTICAL PROPERTIES}

The optical properties of spatially flat Type II lattice models were discussed in [1]. Here we wish to study the optical properties of the Type I models, which have been shown to more faithfully reproduce the correct global dynamics [8, 9].

The equations of motion for null particles in Schwarzschild-de Sitter geometry, as appropriate for this study, are [1]

$$
\begin{aligned}
B & =\left(1-\frac{2 m}{r}-\frac{\Lambda}{3} r^{2}\right) \dot{\tau}+\sqrt{\frac{2 m}{r}+\frac{\Lambda}{3} r^{2}} \dot{r} \\
\dot{r}^{2} & =B^{2}-\frac{J^{2}}{r^{2}}\left(1-\frac{2 m}{r}-\frac{\Lambda}{3} r^{2}\right) \\
\dot{\theta}^{2} & =\frac{J^{2}}{r^{4}}-\frac{J_{\phi}^{2}}{r^{4} \sin ^{2} \theta} \\
\dot{\phi} & =\frac{J_{\phi}}{r^{2} \sin ^{2} \theta}
\end{aligned}
$$

where $B, J$ and $J_{\phi}$ are constants, over-dots denote differentiation with respect to the affine parameter $\lambda$, and $r, \theta$ and $\phi$ are radial and angular Schwarzschild coordinates. The remaining quantities $m$ and $\Lambda$ are the bare mass at the center of each cell and the cosmological constant.

We emphasize that care must be taken in propagating photons between lattice cells, and that it is not appropriate to simply perform a translation of the spatial coordinate system. Rather, one should ensure that in the rest-space of observers at the cell boundary the direction of the photon, and the energy of the photon, is unchanged when leaving the first cell and entering the next. This can be achieved by decomposing the tangent vector to the null rays as

$$
k^{a}=\left(-u_{b} k^{b}\right)\left(u^{a}+n^{a}\right),
$$

where $u^{a}$ is the tangent vector to the trajectory of an observer at the boundary, and $n^{a}$ is a space-like vector that satisfies $n_{a} n^{a}=1$ and $u_{a} n^{a}=0$. The energy of a photon that is measured by this observer is then $E=-u_{a} k^{a}$, and the photon direction is given by the two independent components of $n^{a}$. Matching these three pieces of information at the boundary is sufficient to determine $B$, $J$ and $J_{\phi}$ in the new cell, which completely specifies the trajectory of the photon until it hits another cell boundary, at which point a new matching is performed.

Two methods were proposed in [1] for how to choose the $u^{a}$ that is required for this operation:

Method I: The tangent vector $u^{a}$ is taken to be that of the freely falling observers who follow similar curves to the trajectory of the boundary spheres.

Method II: The tangent vector $u^{a}$ at the cell boundary is taken to be that of the non-geodesic observers who follow the trajectory of the cell boundary at the point where the photons cross it.

The first of these two methods is the least computationally demanding, while the second is constructed to be more accurate. In what follows we will therefore present the results of numerical calculations using Method I, pausing only to make comparisons with Method II in order to gain an estimate of the systematic errors involved. A more detailed description of these two methods is given in [1], to which the reader is referred for details.

In order to calculate optical properties along bundles of null geodesics we use the Sachs equations 10], which in the present situation can be written as [1]

$$
\begin{aligned}
\frac{1}{r_{A}} \frac{d^{2} r_{A}}{d \lambda^{2}}+\sigma^{*} \sigma & =0 \\
\frac{d \sigma}{d \lambda}+\frac{2 \sigma}{r_{A}} \frac{d r_{A}}{d \lambda} & =\frac{3 m J^{2}}{r^{5}} e^{i \Psi},
\end{aligned}
$$

where $r_{A}$ is the angular diameter distance, $\sigma$ is the complex shear scalar, and $\Psi$ is the complex phase of the term driving the shear. As always, the luminosity distance is then given by $r_{L}=(1+z)^{2} r_{A}$ [11], and redshifts are 


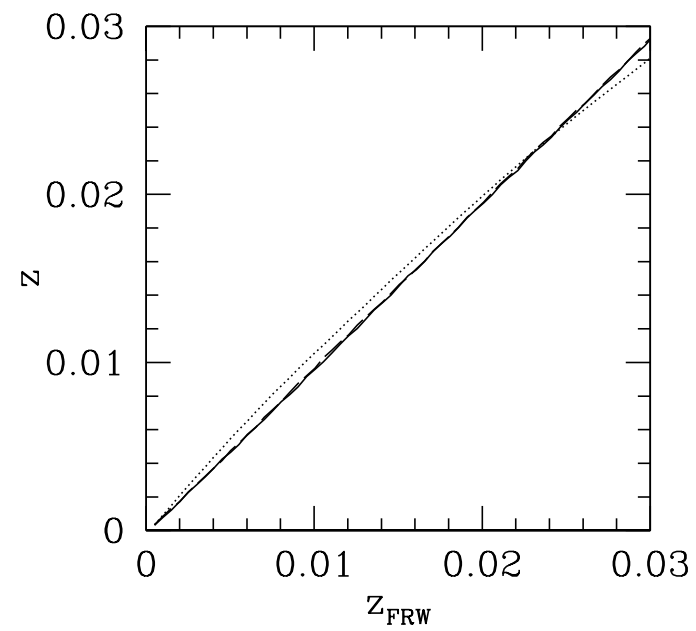

(a) Redshifts along three example trajectories in a Type I model, out to $z \sim 0.03$.

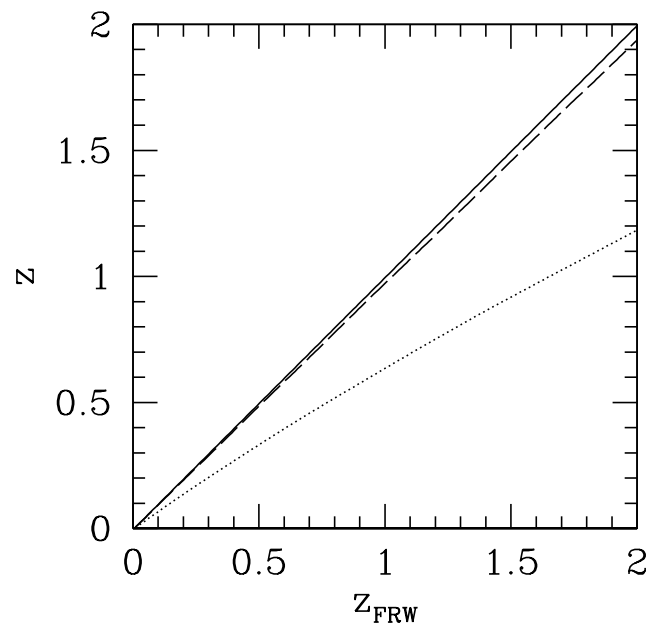

(b) Redshifts in Type I models (dashed line), Type II models (dotted line), and FLRW models (solid line).

FIG. 2: Redshifts in spatially flat LW models as a function of the corresponding redshift in FLRW cosmology.

given by

$$
1+z=\frac{\left(-u^{a} k_{a}\right)_{e}}{\left(-u^{a} k_{a}\right)_{o}}
$$

where observers and sources follow curves with tangent vector $u^{a}$, photons follow curves with tangent vector $k^{a}$, and subscripts $e$ and $o$ denote quantities evaluated at the point of emission and observation of the photons, respectively. In what follows we take $u^{a}$ to be a family of curves that are similar to the trajectory of the boundary sphere of the lattice cell that they are in.

\section{RESULTS}

Let us now consider the results of performing ray tracing through models of Type I. We will first consider cases with $\Lambda=0$, and then cases with $\Lambda \neq 0$.

\section{A. Models with $\Lambda=0$}

With $\Omega_{\Lambda}=0$ the space-time inside each cell is described locally as Schwarzschild geometry. In this case we can propagate null trajectories through the lattice and we find that for Milky Way sized objects separated by $\sim 1 \mathrm{Mpc}$ the resulting redshifts are as displayed in Figure 2. The calculated redshift is shown here as a function of the expected redshift in the corresponding FLRW model.

The right-hand panel in Figure 2 shows redshift, $z$, as a function of the redshift of objects at the same position in the corresponding FLRW model, $z_{F L R W}$. The results of using both Type I and Type II lattice models are displayed in this plot, as well as the line $z=z_{F L R W}$, for comparison. It is clear that the redshifts of distant objects in LW models is strongly dependent on whether Condition I or Condition II is applied. If we parameterize the redshift in these models as

$$
1+z=\left(1+z_{F L R W}\right)^{\langle\gamma\rangle}
$$

then we find that the results of using Condition I are well modeled by $\langle\gamma\rangle \simeq 0.98$, while using Condition II results in $\langle\gamma\rangle \simeq 0.70[1]$. This is a significant difference, with Condition I leading to results that are in much better keeping with those obtained in standard FLRW.

The left-hand panel in Figure 2 shows three individual realizations of photon trajectories in LW models of Type I. The direction of these trajectories is chosen at random, and it can be seen that while they are not identical, they do oscillate around a mean value. This mean is rapidly approached as the distance being observed over is increased, as discussed in detail in [1]. Over the scales plotted in the right-hand panel of Figure2 these trajectories cannot be distinguished by eye. Example trajectories at low $z$ in Type II models are given in [1].

In Figure 3 we show the fractional difference in redshifts calculated using Method I or Method II in models of Type I. It can be seen that these two different methods produce differences in redshift that are at the level of $\lesssim 1 \%$ out to distances that would correspond to $z \sim 2$ in the corresponding FLRW models. This is comparable to the magnitude of the same quantity calculated in Type II models (shown in Figure 8 of [1]), and provides evidence for the validity of using the computationally less expensive Method I when calculating observables in these models at low redshifts.

Integration of the Sachs optical equations in Type I lattice models proceeds in much the same way as the process discussed in [1] for Type II models. The shear that builds 


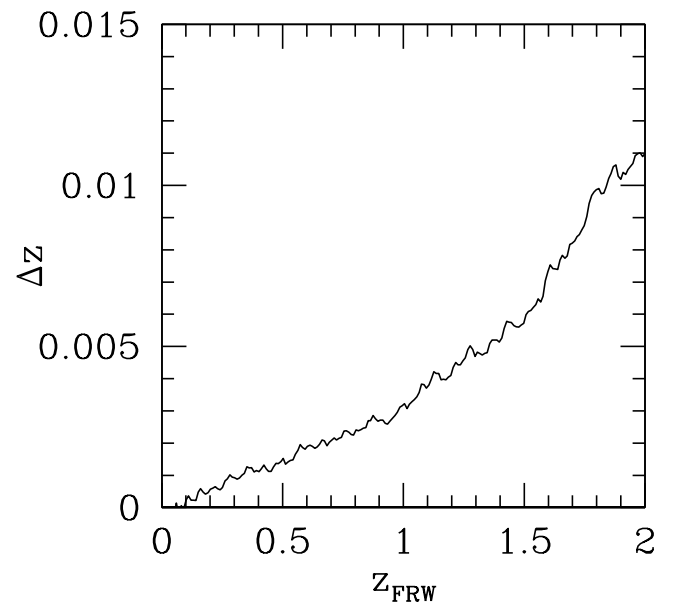

FIG. 3: The fractional difference between redshifts calculated using Method I and Method II in Type I LW models.

in the bundles of null geodesics is still dominated by random events in which the photon trajectories pass close to the central masses, and still performs a random walk. The different condition used to construct the lattice in the present case does not strongly affect this conclusion, as the difference between Condition I and Condition II amounts to a change in the location of the cell boundary. The shearing of the bundles of null geodesics, on the other hand, is dominated by the parts of the photon trajectories that are closest to the cell centers. For Milky Way sized masses separated by $\sim 1 \mathrm{Mpc}$ we find that shear has a negligible affect at low redshifts in models of Type I, as found for models of Type II in [1]. The luminosity distance out to $z \sim 1$ in the present case is then well described by

$$
r_{L} \sim z+0.23 z^{2}+O\left(z^{3}\right)
$$

with a corresponding value for the deceleration parameter of

$$
q_{0} \simeq 0.53
$$

This is a small deviation from the value of $1 / 2$ expected in a spatially-flat, dust-filled FLRW universe with no $\Lambda$, but considerably less than the value of $q_{0} \simeq 1.14$ found in models of Type II [1].

For further discussion of the effects of shear on distance measures, and the formation of caustics, the reader is referred to Section 5 of [1].

\section{B. Models with $\Lambda \neq 0$}

Let us now consider Type I lattice models with $\Omega_{\Lambda} \neq 0$. Models of Type II with $\Omega_{\Lambda} \neq 0$ were considered in [2].

In this case the space-time geometry within each cell is approximated locally as being Schwarzschild-de Sitter.
The first integrals of the equations of motion for null particles in this geometry are given in [2], and the propagation of photons between cells proceeds in a similar fashion to the $\Omega_{\Lambda}=0$ case. The redshift in these situations can again be well modeled by Equation (10), but where the new parameter is now given by $\langle\gamma\rangle=0.98+0.017 \Omega_{\Lambda}^{1.4}$. This function is displayed graphically in Figure 4. The corresponding quantity for Type II models can be found in [2].

Using the redshift relation specified by Equation 10 and Figure 4 we can now calculate distance measures as functions of redshift, as outlined in Section[III. We will be particularly interested in the distance modulus, which is a useful quantity to compare to supernova observations. This is defined as

$$
\Delta \mathrm{dm}=5 \log _{10}\left(\frac{r_{A}}{r_{A}^{\mathrm{Milne}}}\right)
$$

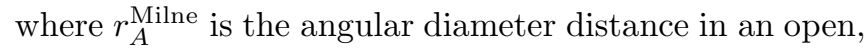
empty Milne universe with the same local Hubble rate. This definition is equivalent to the magnitude of a source, minus the magnitude it would have at the same redshift in a Milne universe.

Fitting the LW models of Type I to the SNLS supernova data [12] we find that the best fitting spatially flat model has $\Omega_{\Lambda}=0.66 \pm 0.05$, while the best fitting spatially flat FLRW model to the same data set has $\Omega_{\Lambda}=0.74 \pm 0.04$. The distance modulus curves for these two models are displayed in Figure 5. The corresponding best fit value using Condition II is $\Omega_{\Lambda}=0.66 \pm 0.04$ [2]. Unlike the redshift relations, we therefore find that the difference in the best fitting value of $\Omega_{\Lambda}$ between LW models constructed using Condition I and Condition II is not large. The fit to the data using Condition I is marginally worse than that found using Condition II, however, with a difference in log likelihood of $|\Delta \ln \mathcal{L}| \simeq 0.1$ between them.

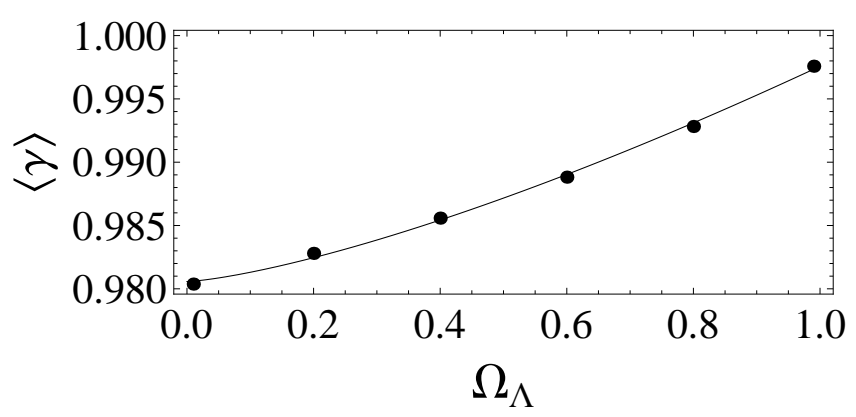

FIG. 4: $\langle\gamma\rangle$ as a function of $\Omega_{\Lambda}$, for models of Type I. These values are much closer to the FLRW limit of $\langle\gamma\rangle=1$ than the models of Type II. 


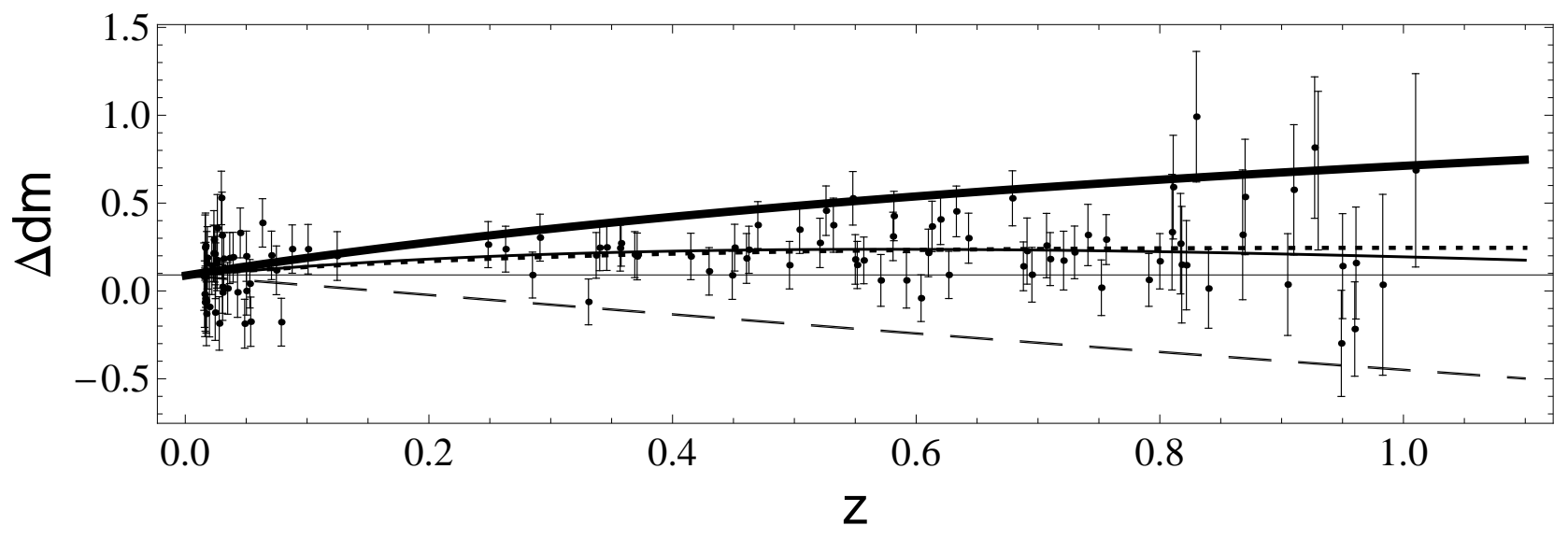

FIG. 5: The best fitting spatially flat LW model of Type I (dotted line), and the best fitting spatially flat FLRW model (thin line). The data represents 115 high and low redshift supernovae from SNLS [12], fitted to the LW model using the SALT light curve fitter [13]. Einstein-de Sitter (dashed line) and de Sitter (thick line) models are also shown, for reference.

\section{DISCUSSION}

In this paper we have considered the optical properties of the Lindquist-Wheeler cosmological models constructed from a lattice of discrete masses. This study builds on previous work that found large deviations from the expected values derived from models that contain a continuous distribution of matter [1, 2].

We find that the way in which the lattice is constructed has considerable consequences for its optical properties. In particular, using Condition I of [3] to construct lattice models results in redshifts that are within a few percent of the expected values from the corresponding FLRW models. This should be compared with differences of $\sim$ $1 / 3$ at redshifts of $z \sim 1$ that were found using Condition II of [3]. Other aspects of the optical properties of these models, however, are less sensitive to the way in which the lattice is constructed. The accumulation of shear along bundles of null geodesics, for example, is largely unaffected. Interestingly, we also find that when $\Lambda$ is included in these models the best fitting value of $\Omega_{\Lambda}$ to supernova data is largely insensitive to the whether we use Condition I or II. In either case $\Omega_{\Lambda}$ is $\sim 10 \%$ less than is required in FLRW cosmology, in order to fit the same data.

Recent studies of cosmological solutions to Einstein's equations that contain discrete masses, rather than a continuous fluid, suggest that the Lindquist-Wheeler models constructed using Condition I provide a good description of the large--scale evolution of a universe filled with regu- larly arranged discrete masses [8, 9]. It even appears that they provide a reasonably good description of the backreaction that such structures should have on the largescale evolution of an initially homogeneous and isotropic space (this effect being large for a small number of very large masses, and small when very many small masses are considered). The present study supports these results by showing that LW lattice models can also accurately reproduce the expected optical properties of a universe filled with many low-mass, discrete, regularly arranged masses [8].

Collectively, this evidence suggests to us that the LW models constructed using Condition I provide a simple and reasonably accurate way to model very highly inhomogeneous distributions of matter [15]. They do this without a priori assuming any background geometry for the Universe, and have been shown to give information that cannot be obtained from exact FLRW solutions alone [9]. The LW models therefore appear to us to be a promising way to investigate the possible effects of nonlinear density fluctuations in relativistic cosmology.

\section{Acknowledgments}

TC and PGF acknowledge the support of the STFC, the BIPAC, and the Oxford Martin School. We are grateful to George Ellis, Kjell Rosquist and Reza Tavakol for helpful discussions and suggestions.
[1] Clifton, T. \& Ferreira, P. G., Phys. Rev. D80, 103503 (2009); erratum Phys. Rev. D84, 109902 (2011).

[2] Clifton, T. \& Ferreira, P. G., JCAP 10, 26 (2009).

[3] Lindquist, R. W. \& Wheeler, J. A., Rev. Mod. Phys. 29,
432 (1957); erratum, Rev. Mod. Phys. 31, 839 (1959).

[4] Wigner, E. P. \& Seitz, F., Phys . Rev. 43, 804 (1933). Wigner, E. P. \& Seitz, F., Phys. Rev. 46, 509 (1934).

[5] Buchert, T., Gen. Rel. Grav. 40, 467 (2008). Clark- 
son, C., Ellis, G. F. R., Larena J. \& Umeh, O., arXiv:1109.2314 (2011).

[6] O'Donnell, K., Optical Properties of the LindquistWheeler Cosmology, Honours project, University of Canterbury, New Zealand (2011).

[7] Uzan, J.-P., Ellis, G. F. R., \& Larena, J., Gen. Rel. Grav. 43191 (2011).

[8] Clifton, T., Class. Quant. Grav. 28, 164011 (2011).

[9] Clifton, T., Rosquist, K. \& Tavakol, R., in preparation.

[10] Sachs, R., Proc. Roy. Soc. Lond. A 264, 309 (1961).

[11] Etherington, I. M. H., Phil. Mag. ser. 7 15, 761 (1933).
[12] Astier, P. et al., Astron. Astrophys. 447, 31 (2006).

[13] Guy, J., Astier, P., Nobili, S., Regnault, N. \& Pain, R., Astron. Astrophys. 443, 781 (2005).

[14] Note that in the original version of [1] it was incorrectly stated that the optical properties of models obeying Condition I were being studied.

[15] This can be contrasted with the LW models constructed using Condition II, which have now been shown to inaccurately reproduce both the required dynamics [8, [9] and the required optics 8$]$. 